\title{
The relationship between perceptual and memorial psychophysics
}

\author{
E. I. CHEW and J. T. E. RICHARDSON \\ Brunel University, Uxbridge, Middlesex UB8 $3 P H$, England
}

\begin{abstract}
Independent groups of subjects made magnitude estimates of the geographical areas of different countries either with a map present or from memory after the map had been studied. Both perceptual and memorial estimates were related to actual area by power functions, in which the memorial exponent was equal to the square of the perceptual exponent. The results provide additional confirmation of a "re-perceptual" model of memorial psychophysics.
\end{abstract}

It is well known that psychophysical judgments of many perceptual characteristics are related to actual physical properties according to a power function of the form $\Psi=k \Phi^{n}$, where $\Psi$ is the psychological magnitude, $\Phi$ is the physical magnitude, $\mathrm{n}$ is a parameter that depends upon the judgment continuum, and $\mathrm{k}$ is a scaling factor (Stevens, 1975). However, it has also been shown that a similar function holds for judgments of remembered quantities (Björkman, Lundberg, \& Tärnblom, 1960; Moyer, Brandley, Sorensen, Whiting, \& Mansfield, 1978; Moyer, Bradley, \& Cutcomb, Note 1). Kerst and Howard (1978) proposed the strong hypothesis that the exponent $\mathrm{n}$ obtained in memorial judgments of a given physical property should be equal to the square of the exponent obtained in perceptual judgments of the same property. In their own experiments, they obtained perceptual and memorial exponents of 1.04 and 1.10 for judgments of visual length and .79 and .60 for judgments of visual area. The results of other studies tended to confirm this relationship for extensive judgment continua, such as length, area, and volume (Moyer et al., 1978), but not for intensive judgment continua, such as brightness and loudness (Moyer et al., Note 1). Kerst and Howard explained their findings in terms of a "re-perceptual" model, according to which two separate transformations relate memorial judgments to actual physical properties: "In an initial perceptual transformation, the physical stimulus arriving at the receptors is processed or transformed into a perceptual experience. It is this transformation that yields the familiar power-function relation between physical magnitude and estimated psychological magnitude. This percept is then stored, in its transformed state, in longterm memory. At recall, a second re-perceptual transformation, very similar or identical to the initial perceptual transformation, is assumed to operate on the stored

The authors are grateful to Sally Davies, Maureen Elstein, Sheila Hornsey, and Liz Patten for their assistance in planning and running the experiment reported here. Requests for reprints should be sent to J. T. E. Richardson, Brunel University, Uxbridge, Middlesex UB8 3PH, England. information to provide a subjective size estimate" (Kerst \& Howard, 1978, p. 331). If the exponents characteristic of the two transformations are assumed to be equal, then this model predicts the obtained relationship between perceptual and memorial judgments.

Kerst and Howard (1978) considered but rejected an alternative hypothesis that attributed the different memorial exponents to uncertainty or forgetting. This would predict that memorial judgments would generally produce lower exponents than would perceptual judgments (cf. Teghtsoonian, 1971), whereas judgments of visual length showed no such trend in Kerst and Howard's research. Nevertheless, one can explain their results by assuming that memorial judgments of area were affected by forgetting, whereas memorial judg. ments of length were not. Indeed, three findings imply that memorial exponents are determined in part by forgetting. First, comparison of Kerst and Howard's Experiments 1 and 2 shows that these exponents were systematically increased when the subjects were allowed 7 min to study the map from which memorial judgments of area subsequently had to be made. Second, as they pointed out, a 24-h retention interval used by Moyer et al. (1978) appeared to cause a decrease in the memorial exponent greater than that predicted by the re-perceptual model. Third, Kerst and Howard's Experiment 1 produced larger exponents with states of the union than with countries of the world, despite the fact that a memorial judgment of geographical area was called for in both cases. Presumably, the reduced familiarity of other countries was a factor here. From the use of confidence ratings, Kerst and Howard found no evidence that memorial judgments were more uncertain than perceptual judgments, but the two groups of subjects might have chosen to distribute their ratings across the (purely subjective and relative) response categories in a similar manner.

This alternative account in terms of uncertainty and forgetting can be directly tested by permitting a greater opportunity for learning before requiring subjects to make memorial judgments of visual area. This should increase the memorial exponent and thus violate the 
relationship between perceptual and memorial psychophysics proposed by Kerst and Howard (1978). The following experiment was therefore an attempt to replicate their Experiment 2 with the following modifications: The number of items to be discriminated was reduced from 48 to 24; the stimuli were chosen to be relatively familiar (countries in Europe or countries that had been mentioned frequently in recent press reports); and the subjects were permitted an indefinite study time before making memorial judgments and were encouraged to pretest the accuracy of their memorial images.

\section{METHOD}

\section{Subjects}

Twenty-four student volunteers participated in the perceptual group and 26 participated in the memorial group.

\section{Materials}

An outline map $(21.0 \times 29.7 \mathrm{~cm})$ was prepared showing the boundaries and names of 24 countries in Europe, Africa, and Asia, selected so as to be relatively familiar to British students. The subjects recorded their size estimates of 23 countries (relative to and excluding France, which was assigned a value of 100 units) on prepared response sheets on which the names of the countries were arranged in a different random order for each participant. The instructions were presented on written forms for both groups, and subjects in the memorial group were additionally given the names of the 24 countries, printed on white index cards, for use in their pretests.

\section{Procedure}

The subjects were tested individually, using instructions and procedures similar to those employed by Kerst and Howard (1978). However, confidence ratings were not elicited. In the memorial task, the subjects were allowed their own time to study the map and to pretest themselves using the cards. They were asked to proceed with the judgment task "when you feel you have memorized the relative sizes of all the 24 countries." Most subjects pretested themselves at least twice, and every subject studied for longer than the 7 min permitted by Kerst and Howard.

\section{RESULTS AND DISCUSSION}

To avoid problems arising from the specific cartographic projection employed, the objective measure of physical size used was the area of the country on the map (calculated by superimposing the map upon graph paper and enumerating the number of squares occupied by each country), rather than the actual geographical area of the country. Geometric mean magnitude estimates were obtained for each country by collapsing across individual subject ratings in each group. Linear correlations were computed between log actual area and log estimates for both individual and group data, and the results of these analyses are shown in Table 1 . Power functions provided a good fit to the data for both perceptual and memorial judgments. The exponent obtained for the perceptual group fell within the range
Table 1

Power Function Exponents (n) and Correlations (r) for Perceptual and Memorial Estimates of Geographical Area

\begin{tabular}{cccccc}
\hline & \multicolumn{2}{c}{ Group Data } & & \multicolumn{2}{c}{ Median Individual Data } \\
& Perception & Memory & & Perception & Memory \\
\hline $\mathrm{n}$ & .79 & .64 & & .84 & .59 \\
$\mathrm{r}$ & .98 & .99 & & .97 & .88 \\
\hline
\end{tabular}

expected from earlier studies. The exponent obtained for the memorial group was substantially larger than that obtained by Kerst and Howard (1978) with no study period, but it was also substantially smaller than that obtained for the perceptual group $[\mathrm{t}(48)=5.08$, $\mathrm{p}<.001]$. In the group data, the memorial exponent was essentially equal to the square of the perceptual exponent (.64 and .63 , respectively). In the individual data, the median memorial exponent was somewhat less than the median square perceptual exponent (.59 vs. $.71)$; this difference was also reflected in the corresponding means (.62 vs. .68), but it was not at all significant $[\mathrm{t}(48)=1.19, \mathrm{p}>.20]$.

This experiment has therefore provided a clear replication of the results obtained by Kerst and Howard (1978), with a greater opportunity for the subjects to retain the information relevant to their memorial decisions. While uncertainty and forgetting clearly do affect performance in this experimental situation, the present study has confirmed the postulated relationship between the exponents obtained in perceptual and memorial judgments. The re-perceptual model proposed by Kerst and Howard appears to be a useful account of memorial psychophysics.

\section{REFERENCE NOTE}

1. Moyer, R. S., Bradley, D. R., \& Cutcomb, S. Memory psychophysics. Paper presented at the annual meeting of the Eastern Psychological Association, Boston, April 1977.

\section{REFERENCES}

BJörkman, M., Lundberg, I., \& Tärnblom, S. On the relationship between percept and memory: A psychophysical approach. Scandinavian Journal of Psychology, 1960, 1, 136-144.

Kerst, S. M., \& Howard, J. H., JR. Memory psychophysics for visual area and length. Memory \& Cognition, 1978, 6, 327-335.

Moyer, R. S., Bradley, D. R., Sorensen, M. H., Whiting, J. C., \& Mansfield, D. P. Psychophysical functions for perceived and remembered size. Science, 1978, 200, 330-332.

Stevens, S. S. Psychophysics: Introduction to its perceptual, neural, and social prospects. New York: Wiley, 1975.

Teghtsoonian, $R$. On the exponents in Stevens' law and the constant in Ekman's law. Psychological Review, 1971, 78, 71-80.

(Received for publication April 23, 1980.) 